\title{
Nondestructive Investigation of Neutron Irradiation Generated Structural Changes of Reactor Steel Material by Magnetic Hysteresis Method
}

\author{
Gábor Vértesy ${ }^{1, *(\mathbb{D}}$, Antal Gasparics ${ }^{1}{ }^{\mathbb{D}}$, Inge Uytdenhouwen ${ }^{2}$, Ildikó Szenthe ${ }^{1}$, \\ Ferenc Gillemot ${ }^{1}$ and Rachid Chaouadi ${ }^{2}$ \\ 1 Centre for Energy Research, H-1121 Budapest, 29-33 Konkoly Thege Miklos ut, Hungary; \\ gasparics.antal@energia.mta.hu (A.G.); szenthe.ildiko@energia.mta.hu (I.S.); \\ gillemot.ferenc@energia.mta.hu (F.G.) \\ 2 SCK CEN Belgian Nuclear Research Centre, 2400 Mol, Belgium; inge.uytdenhouwen@sckcen.be (I.U.); \\ rachid.chaouadi@sckcen.be (R.C.) \\ * Correspondence: vertesy.gabor@energia.mta.hu
}

Received: 23 April 2020; Accepted: 13 May 2020; Published: 15 May 2020

\begin{abstract}
The neutron irradiation embrittlement of four different types of nuclear pressure vessel materials (three base metals and one weld material) were investigated by a magnetic nondestructive testing method, magnetic adaptive testing (MAT). The method is based on the measurement of minor magnetic hysteresis loops on Charpy specimens irradiated by neutrons in the BR2 reactor. Due to the neutron irradiation, the structure of the material was modified. The Charpy impact method is suitable for destructive characterization of material embrittlement. The results of Charpy impact test measurements at SCK CEN Belgian Nuclear Research Centre were compared with the nondestructively measured magnetic parameters. A definite correlation was found between magnetic descriptors and the ductile-to-brittle transition temperature (DBTT), regardless of the type of material or irradiation condition. The results suggest that this "calibration curve" can be used to estimate the DBTT from non-destructive measurements.
\end{abstract}

Keywords: steel degradation; nuclear reactor pressure vessel; magnetic NDT; magnetic adaptive testing; neutron irradiation

\section{Introduction}

Recently, many nuclear power plants having pressurized water vessels operating all over the world were allowed to operate for longer periods than initially foreseen, typically with an additional 20 years of operation. The safe operation of these power plants is essential, and the reactor pressure vessel (RPV) is the most important part of nuclear reactors and their lifetime is determined by the lifetime of RPVs. During service, the pressure vessels are exposed to harsh operation conditions (high temperature and pressure, intensive neutron radiation, etc.), which degrade the properties of pressure vessel materials. It is extremely important to monitor the material properties of RPV to ensure safe operating conditions in conformity with safety requirements. Usually low alloyed CrNiMo or $\mathrm{CrMoV}$ steels are used as RPV materials. These materials are ferromagnetic.

The standard way of monitoring the degradation of reactor pressure vessel materials is the so called surveillance programme. This program uses many samples, which are placed inside the reactor and exposed to neutron irradiation close to the pressure vessel wall. The surveillance specimen sets contain mainly Charpy impact testing samples. The sets of samples are systematically taken out from the reactor and further tested to determine some characteristic parameters. The Charpy impact test is regularly used to characterize structural materials since the beginning of last century [1]. The Charpy 
specimen with a $10 \times 10 \mathrm{~mm}^{2}$ cross-section, a $45^{\circ}$ and $2 \mathrm{~mm}$ deep V-notch and a length of $55 \mathrm{~mm}$ is broken with a swinging hammer and the absorbed energy determined. This energy is measured as a function of test temperature, which allows the deriving of the transition curve. The ductile-to-brittle transition temperature (DBTT) corresponds to the temperature at which the absorbed energy is $41 \mathrm{~J}$. The determination of the DBTT value requires usually several (minimum a dozen) Charpy specimens that should be tested at various temperatures. After neutron irradiation, the Charpy samples become radioactive requiring special safety measures or policies applicable in hot cells.

Nondestructive tests usually do not determine directly the material characteristics, which are measured by destructive tests. It means that before any application of nondestructive tests, they should be very rigorously correlated to the standardized destructive tests. Therefore, before any non-destructive measurements, a calibration is necessary by extensive efforts to validate the methodology. Several non-destructive methods are available. One of them is the method based on the Seebeck effect. It was found $[2,3]$ that by very precise measurement of the Seebeck coefficient, the non-destructive inspection of the neutron irradiation generated embrittlement of RPV steels was possible. The ultrasonic technique is another widely and successfully used, standardized method for the detection of material degradation [4-6], but till now this technique has not been able to detect the neutron irradiation generated embrittlement of reactor steel.

Magnetic methods play an important role in electromagnetic nondestructive evaluation $[7,8]$. One of them, the Barkhausen noise (MBN) method has been developed as a useful technique for investigation of surface defects caused from manufacture, microstructure change and residual stresses [9-12]. Another technique, magnetoacoustic emission, can be used for the monitoring or determination of residual stresses [13].

The analogy between modification of material microstructure due to different effects (i.e., how dislocations move) and modification of magnetic behavior under a magnetizing field (i.e., how magnetic domain walls move) can be used to characterize the materials by magnetic hysteresis measurements. Domain walls and move of dislocations are both influenced by the defects in the micro-structure when they move in a ferromagnetic material. The correlation between magnetic and mechanical hardness in ferromagnetic materials is well-known and well understood [14,15]. An advantage of magnetic methods is that they are technically simple, the technique is not expensive and it can be applied via electrical cables and coils. It means that the control electronic device can be put outside of the protected area where the radioactive specimens must be kept. Applicability of magnetic methods for quantitative determination of material degradation have been proven by several successful investigations. For instance, the influence of thermal treatment [16], effect of cold rolling [17], influence of neutron irradiation on reactor pressure vessel steels and welds were investigated [18-20].

A relatively novel and promising method of non-destructive evaluation, based on magnetic hysteresis measurements, is the so-called magnetic adaptive testing (MAT) [21]. In this method series of minor magnetic hysteresis loops are measured in contrast to the major loop measurement. Parameters are calculated from the points of minor loops characterizing the material degradation. As it has been proven [22], MAT is a sensitive, robust and multi-parametric method. Three different series of reactor steel material samples, irradiated by neutrons, were investigated by MAT in our previous work [reference]. In all cases, good correlation was found between magnetic descriptors and the neutron fluence [23]. Applicability of MAT is presented in this work for four different RPV steel materials by comparing the destructively measured transition temperature (DBTT) values with the non-destructively determined magnetic descriptors.

\section{Sample Preparation and Processing}

Charpy specimens with standard geometry and dimensions (shown in Figure 1) from 22NiMoCr37, A508-B and HSST-03 base metals and of 18MND5-W weld material were studied. These materials belong to the typical RPV steel groups (western RPV design). The chemical compositions measured by optical emission spectroscopy of the four materials are presented in Table 1. 


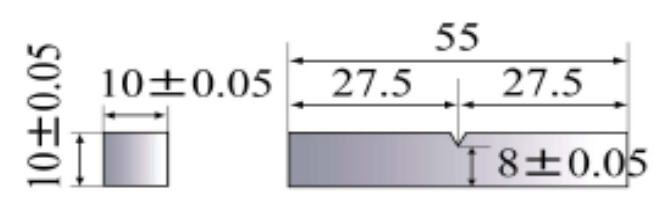

Figure 1. Shape and dimensions (in $\mathrm{mm}$ ) of the measured samples. (Each sample had a V-notch at one face.).

Table 1. Chemical compositions (wt $\%$ ) of the investigated materials by optical emission spectroscopy.

\begin{tabular}{cccccccccc}
\hline Material & C & Si & Mn & P & S & Cr & Mo & Ni & Cu \\
\hline 18MND5-W & 0.09 & 0.23 & 1.21 & 0.018 & 0.009 & 0.12 & 0.49 & 0.96 & 0.13 \\
\hline 22NiMoCr37 & 0.20 & 0.25 & 0.87 & 0.009 & 0.007 & 0.39 & 0.49 & 0.85 & 0.06 \\
\hline A508-B & 0.20 & 0.31 & 1.40 & 0.010 & 0.008 & 0.10 & 0.45 & 0.74 & 0.06 \\
\hline HSST-03 & 0.25 & 0.27 & 1.42 & 0.013 & 0.011 & 0.13 & 0.48 & 0.62 & 0.12 \\
\hline
\end{tabular}

The samples were irradiated in the CALLISTO loop of the BR2 reactor at different temperatures ranging between 150 and $305{ }^{\circ} \mathrm{C}$ by fast neutrons ( $\mathrm{E}>1 \mathrm{MeV}$ energy) to a total neutron fluence range of $2.66 \times 10^{19}-9.36 \times 10^{19} \mathrm{n} / \mathrm{cm}^{2}$ [24] in the CHIVAS irradiation campaigns. Since irradiated samples are radioactive, they must be measured only in hot cell laboratories. Charpy impact testing standard (ASTM-23-16b, [25]) was used to get the $41 \mathrm{~J}$ ductile-to-brittle transition temperature (DBTT). A calibrated impact tester (300 J MFL PMT PSW300M type, made by (Maschinenfabrik Liezen und Gießerei Ges.m.b.H. Werkstraße 5, 8940 Liezen, Austria) was applied for the testing with ISO tup. Samples were cooled down in an environmental chamber with controlled temperature. All tests were performed according prevailing standards, namely E23 and E2215. DBTT values were determined for groups of samples irradiated by the same neutron fluence. The uncertainty on DBTT is usually not exceeding $+/-10^{\circ} \mathrm{C}$, except on heterogeneous materials.

Within this experimental series 3 pieces of not irradiated (reference) and 12 pieces of irradiated sample were measured made of $22 \mathrm{NiMoCr} 37$ type base material, 3 pieces of not irradiated and 8 pieces of irradiated sample were measured made of HSST- 03 base type material, 5 pieces of not irradiated and 7 pieces of irradiated sample were measured made of A508-B base type material, and 5 pieces of not irradiated and 7 pieces of irradiated sample were measured made of $18 \mathrm{MND} 5-\mathrm{W}$ weld material.

\section{Magnetic Adaptive Testing}

Minor magnetic hysteresis loops are measured systematically by this technique. A complex set of minor hysteresis loops contains all information about the material's microstructure modification generated by external effects. The theoretical Preisach hysteresis model [26] describes this phenomenon. Measurement starts from a zero magnetizing field after careful demagnetization of the sample by decreasing AC magnetizing current. The AC magnetizing field is step by step increased during the measurement up to its saturation point. A special measuring device, the permeameter, was designed and built for this purpose [27], which is controlled by a PC. Samples to be measured are magnetized by a magnetizing yoke, which is placed on the sample. The measurement is performed on the surface opposite to the V-notch. The magnetizing yoke is a half transformer core made of laminated Fe-Si sheets. It contains two coils, an exciting coil and a pick-up coil, both of them are wound on yoke legs. The yoke size should be chosen such as to accommodate the sample size. The possible largest volume should be magnetized, but the yoke legs should not be longer/wider than the surface of the sample. In the case of the presently measured samples, the cross-section of the yoke leg is $10 \times 5 \mathrm{~mm}^{2}$. The sample holder designed for hot cell measurements can be seen in Figure 2. Below the sample is the magnetizing yoke, and the replacement of samples can be done easily by a manipulator. Control of the measurement is performed by an electronic unit, which is located outside of the hot cell. 


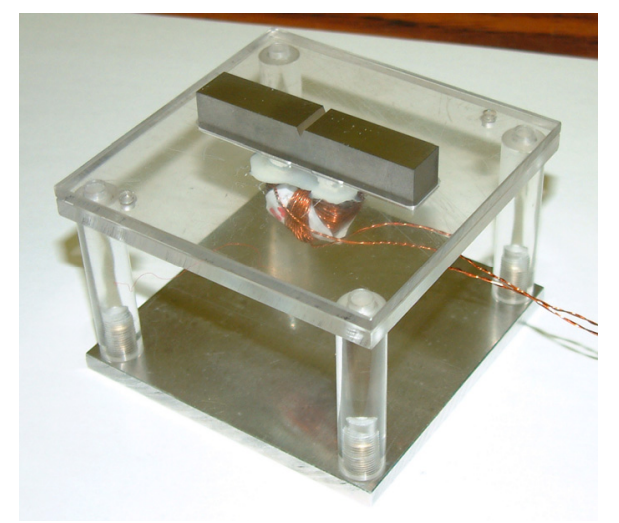

Figure 2. Photo of the sample holder. A Charpy sample is placed on the top V-notch is opposite to the magnetizing yoke.

Magnetization of the sample is made by a triangular waveform current with a step by step increasing amplitude producing a triangular time variation of the magnetizing field. The slope of the magnetizing current is fixed. A signal generated in the pick-up coil is proportional to the differential permeability of the sample if the magnetizing field variation is linear with time.

An evaluation program was developed for processing the measured data. Square grate of elements are generated and experimental raw data are interpolated into this grate. This is the $\mu$ or permeability matrix, which is the base of any further evaluation. The elements of the matrix are characterized by $\mu \equiv \mu\left(h_{a}, h_{b}\right)$ values, where $h_{b}$ is the minor loop amplitude and $h_{a}$ is the magnetizing field. These $\mu$-elements are designated as "MAT-descriptor" and they characterize the material structure variation of the investigated specimens.

Another evaluation program was developed for processing of the matrices. The $\mu \equiv \mu\left(h_{a}, h_{b}\right)$ matrix elements are normalized by the same element of the reference matrix. In this process, $\mu(x)$-degradation functions are calculated. These functions characterize the degradation of material. In the present work, $x$ is the transition temperature, i.e., DBTT. The whole MAT procedure is described in details in Ref. [22].

The magnetizing yoke, the sample itself and the air gap between sample surface and the sole of magnetizing yoke form an open magnetic circuit. Due to the air gap, part of the magnetic flux is scattered to the air, this part is not effective from the point of view of sample's magnetization. In other words, the measured permeability decreases significantly proportionally with the stray field, it is not a bulk property of the measured sample any more. Consequently, the surface condition (surface irregularity or roughness, surface corrosion) plays a substantial role on the shape of the investigated permeability curves and on the maximal measured permeability. Furthermore, any pollution and dust on the surface can modify surface conditions. These unwanted effects decrease the measured permeability. Considering that MAT descriptors ( $\mu$-degradation functions) are calculated from the permeability, they are very much influenced by surface conditions. If the surface roughness is the same or similar for all samples within the same experimental series of samples, the quality of surface does not play role, because MAT descriptors are normalized by the reference sample's descriptor, and only the difference between samples behavior is determined.

However, due to neutron irradiation, it happens frequently that the surface roughness varies from one sample to another, and this can cause a problem. To get reliable results during magnetic measurements, the harmful influence of the surface roughness was reduced by fine polishing of the surface of the specimens in the hot cell before all measurements. Polishing was done through several minutes of grinding by a " 1200 " code fine $\mathrm{SiC}$ polishing paper. This polishing improves significantly the surface quality, which can be observed on the detected permeability curves. 


\section{Results and Discussion}

All the four series of the above described Charpy specimens made of different reactor vessel materials were measured by the MAT method before and after neutron irradiation. The $\mu$ matrix elements, which characterize the material degradation, were calculated from the measured permeability loops. There are many (hundreds or thousands, depending on the evaluation) matrix elements, and the so called sensitivity map helps to find the most suitable (optimal) degradation functions. The sensitivity map is a $3 \mathrm{D}$ plot, and it is calculated during the data processing. It illustrates the relative sensitivity of the $\mu(x)$-degradation functions, as represented by the value of the slope of the linear regression computed for each $\mu(x)$ and plotted into the sensitivity map at the position of the relevant coordinates $\left(h_{a}, h_{b}\right)$. The most responsive part of the sensitivity matrix of the investigated $22 \mathrm{NiMoCr} 37$ samples is shown, as an illustration, in Figure 3, as the red area. It is seen that within the area of top sensitivity the $\mu\left(h_{a}, h_{b}\right)$-degradation functions vary only very slightly from one another, so the neighboring elements provide practically the same value. The crossed lines in Figure 3 indicate the position of the $\left(h_{a}, h_{b}\right)$-coordinates $\left(h_{a}=75 \mathrm{~mA}, h_{b}=110 \mathrm{~mA}\right)$, which determines the optimal $\mu$-degradation function with respect to the independent parameter (DBTT). The neighboring points would result in the same value, however. This makes the choice of the proper, sensitive (optimal) descriptor to be very reliable and well reproducible. Sensitivity matrices were calculated and used for all sample series, but for other materials it is not shown here. The position of the optimal $\left(h_{a}, h_{b}\right)$-coordinates depends on the material, it is not the same for all sample series.

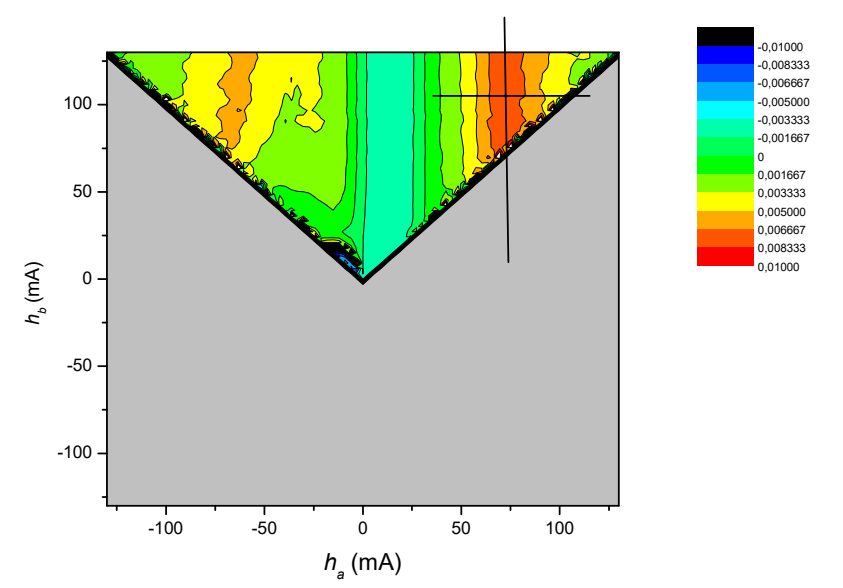

Figure 3. Map of relative sensitivity of the $\mu$-degradation functions, $\mu(D B T T) \equiv \mu\left(h_{a}, h_{b}\right)$. The crossing point of the lines indicate the field coordinates of the $\mu_{i}(D B T T)$-degradation function used for samples of $22 \mathrm{NiMoCr} 37$ material.

These matrix elements of irradiated samples were normalized by the same $\mu$ matrix elements of the reference (not irradiated) sample within each types of material. In such a way, these magnetic parameters reflect directly the modification of the material properties with respect to the reference sample. These normalized parameters were compared with the independently measured DBTT values. The DBTT value from destructive Charpy impact tests comes from the temperature value at $41 \mathrm{~J}$ impact energy $\left(T_{41} \mathrm{~J}\right)$. This curve is made with minimum of 6 to 8 Charpy samples. The optimally chosen MAT descriptor is on each Charpy sample separately. The results of the evaluation are shown in Figures 4-7 for each material. During the evaluation, the optimal MAT descriptors were determined, as explained above. In the graphs each point represents a different sample. The same DBTT value belongs to more than one samples.

The low number and the big scatter of measured points make questionable any rigorous mathematical fitting, as the main point of fitting is just to "lead the eye". A linear fit was performed in each graph, because according to our previous results and literature data this type of correlation was expected. 


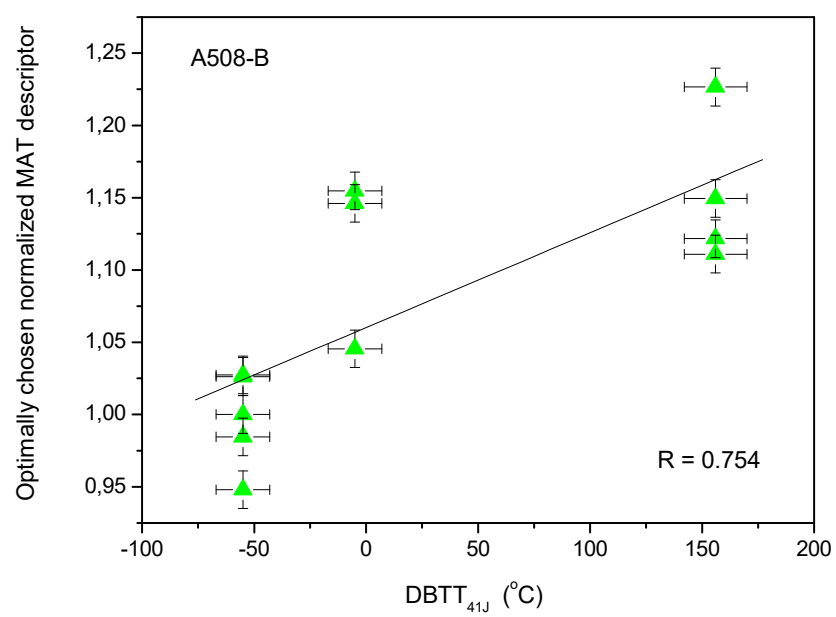

Figure 4. Optimally chosen magnetic adaptive testing (MAT) descriptor of reference and of irradiated A508-B type reactor steel base material as a function of transition temperature, ductile-to-brittle transition temperature (DBTT).

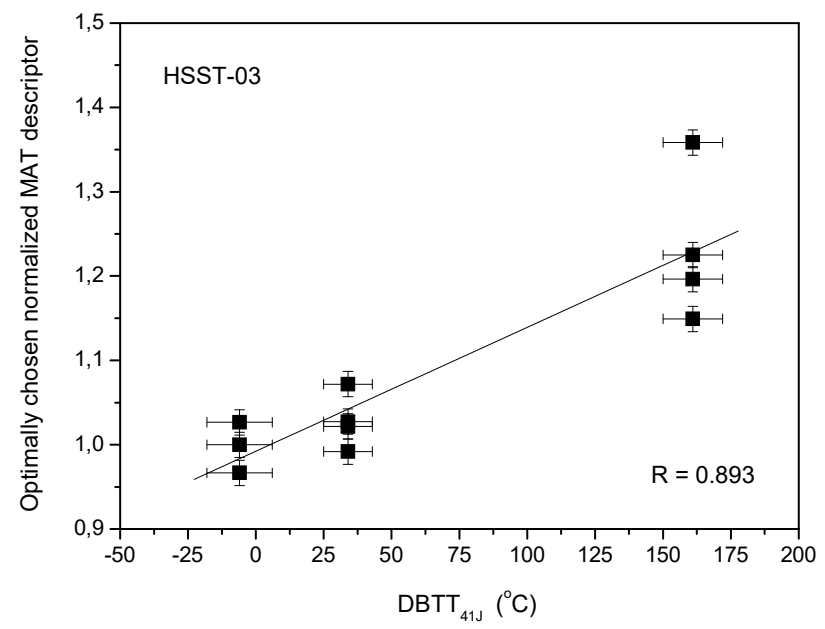

Figure 5. Optimally chosen MAT descriptor of reference and of irradiated HSST-03 type reactor steel base material as a function of transition temperature, DBTT.

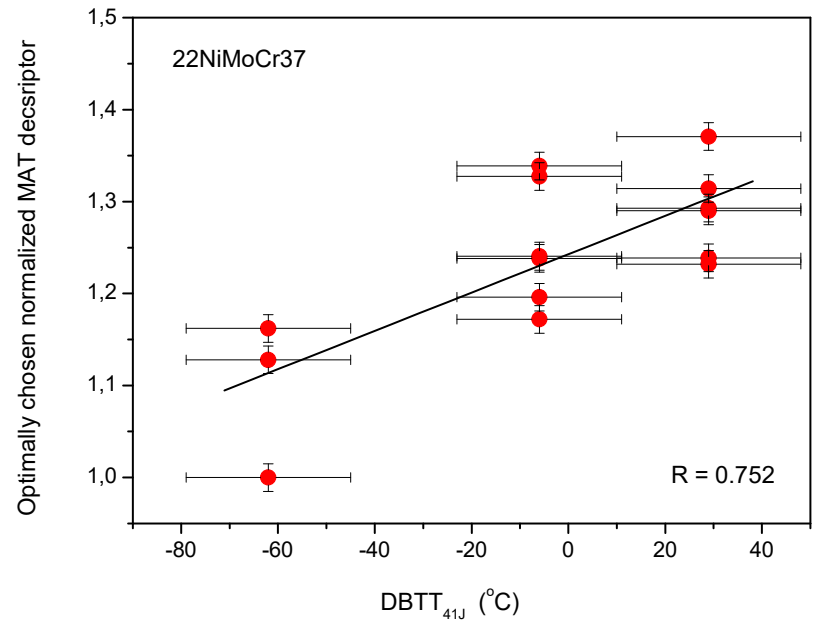

Figure 6. Optimally chosen MAT descriptor of reference and of irradiated 22NiMoCr37 type reactor steel material as a function of transition temperature, DBTT. 


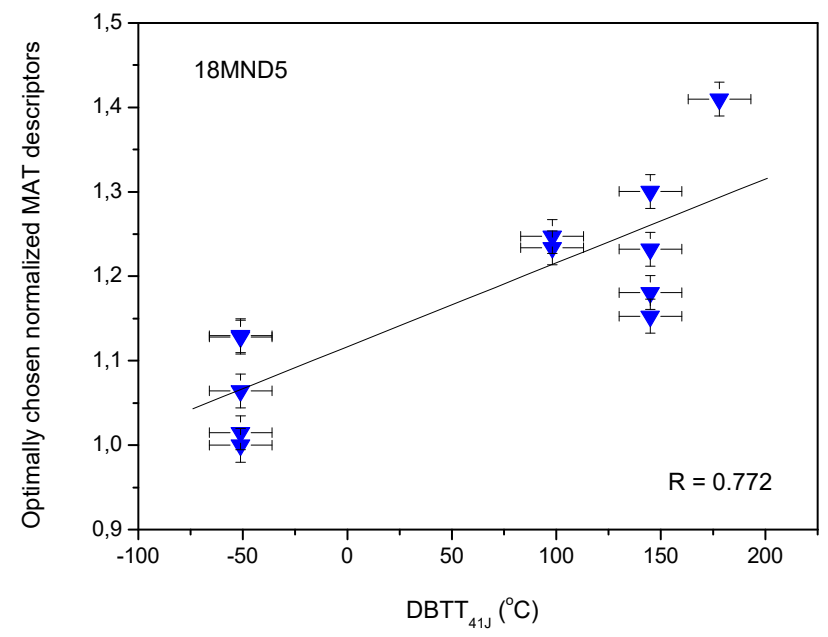

Figure 7. Optimally chosen MAT descriptor of reference and of irradiated 18MND type weld material as a function of transition temperature, DBTT.

As it is seen in Figures 4-7, a linear or closely linear correlation can be observed in all four types of materials between MAT descriptor and transition temperature. The best fitting was obtained by linear fit indicated in the figures together with the value of regression factor, $R$. These results were expected on the basis of our previous experience and other literature data, e.g., $[12,20,23]$. The graphs above show how the magnetic descriptors are modified by neutron irradiation. This ratio, the normalized MAT descriptor at the maximum DBTT value is typically $30-40 \%$, with respect to the reference (not irradiated) samples. This change in magnetic parameters seems to be large enough for a reliable and sensitive detection of neutron irradiation damage generated in the material.

On the other side, it is evident that the scatter of points is rather large. Each sample was measured several times after removing and replacing back the sample. Error bars represent the repeatability of the measurement. As clearly seen, repeated measurements of the same sample resulted in much less error than the scatter of points (results from different samples) in the above graphs. The samples were machined from the same material and samples having the same DBTT value were irradiated by the same neutron fluence, so material properties should be rather close to each other. The large scatter between different samples can be probably attributed mainly to the different surface conditions, which existed even after polishing the surface. We tried to polish different samples similarly, but this light polishing was not enough to homogenize the surface roughness of the different samples.

The main limitation of the method is that it is at least as sensitive to surface condition as to irradiation damage. But in spite of this fact, by measuring several samples with the same material degradation having different surface conditions, a reliable calibration curve can be determined, as presented in the above figures. In future, additional measurements will be performed to reduce the influence of the surface roughness by inserting a suitable nonmagnetic spacer between the sample surface and magnetizing yoke. This assumption is based on preliminary observations of the reduced influence of surface conditions when inserting an appropriate nonmagnetic spacer. In such a way, the samples' degradation with different surface roughnesses can be reliably inspected $[28,29]$.

By using the experimental results, an estimation can be done for the transition temperature. This estimation was made as explained in Figure 8. The linear fitting was used to determine the estimated DBTT value. This graph is just for the illustration of the method; similar procedure was performed on all measured points of all materials. First the result of the measurement, e.g., optimally chosen MAT descriptors vs. transition temperature were illustrated. This graph is identical with Figure 6, only the MAT descriptors are given not in normalized way. Then a linear fitting was applied. After it the magnetic values were considered for each sample. (In this graph one of the samples was chosen to show the procedure. It appears as the red circle, while measured points of other samples are 
represented by black squares. The estimated DBTT value for this sample was calculated as shown in the figure. It is seen very well that large difference can be between measured and estimated DBTT values. This difference is caused by the scatter of points.

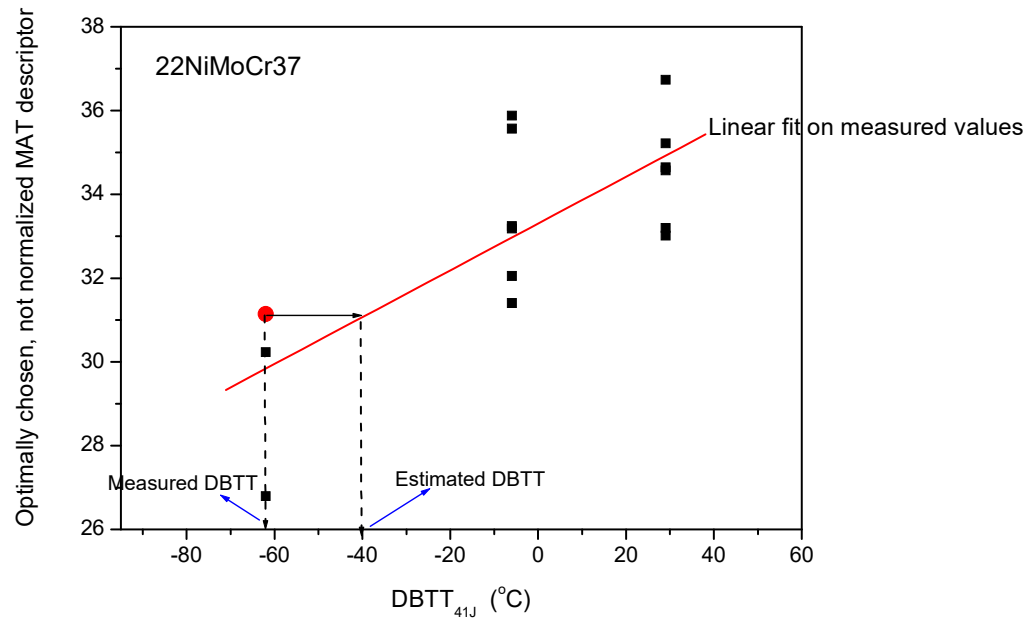

Figure 8. Optimally chosen, not normalized MAT descriptor of reference and of irradiated 22NiMoCr37 type reactor steel material as a function of transition temperature, DBTT. The procedure of how the estimated DBBT value was determined is shown on one of the samples (red circle).

By applying the above demonstrated procedure, the correlations between the measured and estimated values of the transition temperature were determined for all measured samples. It is also possible to consider these correlations within one graph, regardless of the type of material. This is shown in Figure 9. A clear, linear or closely linear tendency was observed. It is worthy of mentioning that this correlation does not depend on the actual material. The slope of the linear fitting is 1.0217 with 0.106 error and the regression is 0.843 . In an ideal case, evidently, the slope of correlation between the measured and estimated values of the parameter should be 1. The experienced 1.0217 slope is rather close to the expected value, and the regression is also not bad.

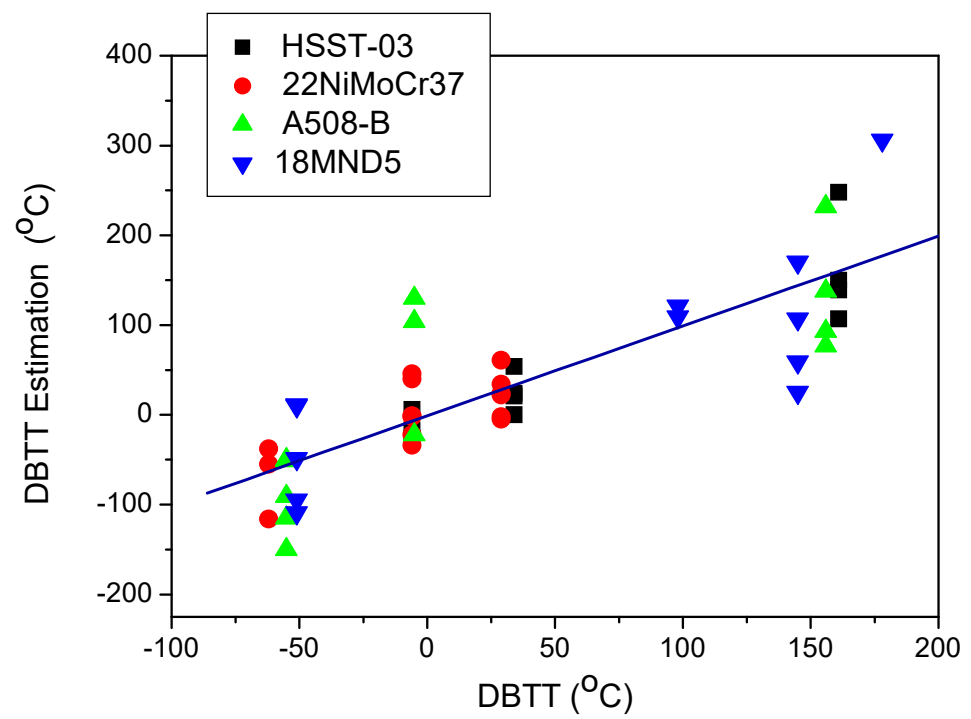

Figure 9. Estimated DBTT values as functions of measured DBTT values for all investigated materials.

It means that the calculated linear correlation can probably be successfully used for estimation of the transition temperature, even in the case of the not measured samples. Considering the huge scatter of points the detected linear correlation is surprisingly good. This is the most important 
message of the present work. We are aware that the number of points is low for making really good statistics, but anyway, as a first attempt, this result is promising. For approval of this result, many more measurements should be done in the future, and the surface roughness problem should be handled properly.

The magnetic non-destructive methods do not sense the neutron fluence. Instead, their response is in correlation with the structure changes of the material caused by the neutron irradiation. According to the adopted standards, the complex mechanical material properties are characterized by only a few measureable parameters such as the solidity, which is expressed in terms of ductile-to-brittle transition temperature value. The shape of the transition temperature function is known, therefore the standard defines at least seven of its significant points to be measured for the identification. Considering the Charpy impact test, in fact, approximately fifty individual tests are required for the reliable DBTT estimation. This indicates significant scattering of the DBTT values. Typically, the scattering of the DBTT values are determined by the Charpy impact test can reach the $+/-20^{\circ} \mathrm{C}$. Due to the nature of the Charpy impact test method, the DBTT value cannot be determined for a single specimen individually, but it can be derived for the set of them. However, this cannot lead to the conclusion that they are identical for all of the specimens of the set due to the material inhomogeneity. Therefore, the scattering of the nondestructively measured (NDT) data cannot be analyzed on a limited number of specimens, since it is overlapped with the scattering of the DBTT values. From the application point of view, the NDT method should not provide the estimates of the DBTT values with infinite precision. Instead, the required resolution is defined as $50{ }^{\circ} \mathrm{C}$. In order to be able to prove this, a large set of specimens has to be studied and the overlapping scatterings of the DBTT values and NDT results have to be separated. This is beyond to the framework of this paper.

\section{Conclusions}

Destructively measured ductile-brittle transition temperature values were compared with the nondestructively measured magnetic parameters, measured by minor magnetic hysteresis loops on different neutron irradiated reactor pressure vessel materials. In spite of the large scatter of points-which is due to the different surface conditions of the samples-good correlation was found between these characteristics. Magnetic adaptive testing can be a promising method. This type of magnetic measurement can also be easily applied for radioactive materials. Another significant advantage of the method is that it is not necessary to magnetically saturate the samples, which is a very difficult task in practical measurements.

A clear trend of the magnetic descriptor is observed even when many measurements of specimens with known fluency are performed, whereas the estimation of the DBTT based on a single measurement on a sample with unknown fluency will result in a large uncertainty. To measure a set of samples with the same fluency reduces the uncertainty significantly, which would be possible within a surveillance program where several specimens with the same fluency can be measured.

Author Contributions: Conceptualization, investigation, methodology and formal analysis, G.V.; investigation, G.V., I.S., F.G., I.U. and R.C.; project administration, A.G. and I.S. All authors have read and agreed to the published version of the manuscript.

Funding: This research was supported by “NOMAD" project. This project (Nondestructive Evaluation System for the Inspection of Operation-Induced Material Degradation in Nuclear Power Plants) received funding from the Euratom research and training programme 2014-2018 under grant agreement No. 755330.

Conflicts of Interest: The authors declare no conflict of interest. 


\section{References}

1. Ferreño, D.; Gorrochategui, I.; Gutiérrez-Solana, F. Degradation Due to Neutron Embrittlement of Nuclear Vessel Steels: A Critical Review About the Current Experimental and Analytical Techniques to Characterise the Material, with Particular Emphasis on Alternative Methodologies. In Nuclear Power-Control, Reliability and Human Factors; Tsvetkov, P., Ed.; IntechOpen Limited: London, UK, 2011; ISBN 9789533075990. Available online: http://www.intechopen.com/articles/show/title/non-destructivetesting-for-ageing-management-of-nuclear-power-components (accessed on 14 May 2020).

2. Niffenegger, M.; Leber, H.J. Monitoring the embrittlement of reactor pressure vessel steels by using the Seebeck coefficient. J. Nucl. Mater. 2009, 389, 62. [CrossRef]

3. Niffenegger, M.; Reichlin, K.; Kalkhof, D. Application of the Seebeck effect for monitoring of neutron embrittlement and low-cycle fatigue in nuclear reactor steel. Nucl. Eng. Des. 2005, 235, 1777-1788. [CrossRef]

4. Seiler, G. Early Detection of Fatigue at Elevated Temperature in Austenitic Steel Using Electromagnetic Ultrasound Transducers. In Proceedings of the Seventh International Conference on Low Cycle Fatigue: LCF7, Deutscher Verband für Materialforschung und-prüfung e.V. (DVM), Aachen, Germany, 9-11 September 2013; p. 359.

5. Smith, R.L.; Rusbridge, K.L.; Reynolds, W.N.; Hudson, B. Ultrasonic attenuation, microstructure and ductile to brittle transition temperature in Fe-C alloys. Mater. Eval. 1993, 41, 219-222.

6. Dobmann, G.; Kröning, M.; Theiner, W.; Willems, H.; Fiedler, U. Nondestructive characterization of materials (ultrasonic and magnetic techniques) for strength and toughness prediction and the detection early creep damage. Nucl. Eng. Des. 1995, 157, 137-158. [CrossRef]

7. Jiles, D.C. Magnetic Methods in Nondestructive Testing. In Encyclopedia of Materials Science and Technology; Buschow, K.H.J., Ed.; Elsevier Press: Oxford, UK, 2001; p. 6021.

8. Blitz, J. Electrical and Magnetic Methods of Nondestructive Testing; Adam Hilger IOP Publishing, Ltd.: Bristol, UK, 1991.

9. Lo, C.C.H.; Jakubovics, J.P.; Scrub, C.B. Non-destructive evaluation of spheroidized steel using magnetoacoustic and Barkhausen emission. IEEE Trans. Magn. 1997, 33, 4035-4037. [CrossRef]

10. Kikuchi, H.; Ara, K.; Kamada, Y.; Kobayashi, S. Effect of microstructure changes on Barkhausen noise properties and hysteresis loop in cold rolled low carbon steel. IEEE Trans. Magn. 2009, 45, 2744-2747. [CrossRef]

11. Hartmann, K.; Moses, A.J.; Meydan, T. A system for measurement of AC Barkhausen noise in electrical steels. J. Magn. Magn. Mater. 2003, 254-255, 318-320. [CrossRef]

12. Pirfo Barroso, S.; Horváth, M.; Horváth, Á. Magnetic measurements for evaluation of radiation damage on nuclear reactor materials. Nucl. Eng. Des. 2010, 240, 722-725. [CrossRef]

13. Augustyniak, B.; Chmielewski, M.; Piotrowski, L.; Kowalewski, Z. Comparison of properties of magnetoacoustic emission and mechanical barkhausen effects for P91 steel after plastic flow and creep. IEEE Trans. Magn. 2008, 44, 3273-3276. [CrossRef]

14. Devine, M.K. Magnetic detection of material properties. J. Min. Met. Mater. JOM 1992, 44, 24-30. [CrossRef]

15. Kronmüller, H.; Fähnle, M. Micromagnetism and the Microstructure of Ferromagnetic Solids; Cambridge University Press: Cambridge, UK, 2003.

16. Tomáš, I.; Kadlecová, J.; Konop, R.; Dvořáková, M. Magnetic Nondestructive Indication of Varied Brittleness of 15Ch2MFA Steel. In Proceedings of the 9th International Conference on Barkhausen Noise and Micromagnetic Testing (ICBM9), Hejnice, Czech Republic, 28-29 June 2011; pp. 55-63. ISBN 978-952-67247-4-4 (paperback), ISBN 978-952-67247-5-1 (CD-ROM).

17. Takahashi, S.; Kobayashi, S.; Kikuchi, H.; Kamada, Y. Relationship between mechanical and magnetic properties in cold rolled low carbon steel. J. Appl. Phys. 2006, 100, 113908. [CrossRef]

18. Takahashi, S.; Kikuchi, H.; Ara, K.; Ebine, N.; Kamada, Y.; Kobayashi, S.; Suzuki, M. In situ magnetic measurements under neutron radiation in Fe metal and low carbon steel. J. Appl. Phys. 2006, 100, 023902. [CrossRef]

19. Dobmann, G.; Altpeter, I.; Kopp, M.; Rabung, M.; Hubschen, G. ND-Materials Characterization of Neutron Induced Embrittlement in German Nuclear Reactor Pressure Vessel Material by Micromagnetic NDT Techniques. In Electromagnetic Nondestructive Evaluation (XI); IOS Press: Amsterdam, The Netherlands, 2008; p. 54. ISBN 978-1-58603-896-0. 
20. Kobayashi, S.; Gillemot, F.; Horváth, Á.; Székely, R. Magnetic properties of a highly neutron-irradiated nuclear reactor pressure vessel steel. J. Nucl. Mater. 2012, 421, 112-116. [CrossRef]

21. Tomáš, I. Non-destructive magnetic adaptive testing of ferromagnetic materials. J. Magn. Magn. Mater. 2004, 268, 178-185. [CrossRef]

22. Tomáš, I.; Vértesy, G. Magnetic Adaptive Testing. In Nondestructive Testing Methods and New Applications; Omar, M., Ed.; IntechOpen Limited: London, UK, 2012; ISBN 978-953-51-0108-6. Available online: http: //www.intechopen.com/articles/show/title/magnetic-adaptive-testing (accessed on 14 May 2020).

23. Tomáš, I.; Vértesy, G.; Gillemot, F.; Székely, R. Nondestructive magnetic adaptive testing of nuclear reactor pressure vessel steel degradation. J. Nucl. Mater. 2013, 432, 371-377. [CrossRef]

24. Uytdenhouwen, I.; Chaouadi, R.; Rabung, M. NOMAD: Non-Destructive Evaluation (NDE) System for the Inspection of Operation-Induced Material Degradation in Nuclear Power Plants-Overview of the Neutron Irradiation Campaigns. In Proceedings of the Pressure Vessel and Piping Conference ASME 2020-PVP2020-21512, Minneapolis, MN, USA, 19-24 July 2020.

25. DeVan, M.J.; Lowe, A.L.; Hall, J.B. Fracture toughness test results of thermal aged reactor vessel materials. ASTM Spec. Technical Publ. 1996, 1270, 660-669.

26. Mayergoyz, I.D. Mathematical Models of Hysteresis; Springer: New York, NY, USA, 1991.

27. Tomáš, I.; Perevertov, O. Permeameter for Preisach Approach to Materials Testing. In JSAEM Studies in Applied Electromagnetics and Mechanics, 9th ed.; Takagi, T., Uesaka, M., Eds.; IOS Press: Amsterdam, The Netherlands, 2001; p. 5.

28. Vértesy, G.; Gasparics, A.; Uytdenhouwen, I.; Chaouadi, R. Influence of surface roughness on non-destructive magnetic measurements. Glob. J. Adv. Eng. Technol. Sci. 2019, 6, 25-33.

29. Tomáš, I.; Kadlecová, J.; Vértesy, G. Measurement of flat samples with rough surfaces by magnetic adaptive testing. IEEE Trans. Magn. 2012, 48, 1441-1444. [CrossRef]

(C) 2020 by the authors. Licensee MDPI, Basel, Switzerland. This article is an open access article distributed under the terms and conditions of the Creative Commons Attribution (CC BY) license (http://creativecommons.org/licenses/by/4.0/). 